\title{
Diseño de escenarios de aprendizaje con interfaces naturales y realidad aumentada para apoyar la inclusión de estudiantes con discapacidad auditiva en la educación media superior
}

\author{
Carmen Cerón, Etelvina Archundia, Alfonso Garcés, Beatriz Beltrán, Jair Migliolo \\ Benemérita Universidad Autónoma de Puebla, \\ Facultad de Ciencias de la Computación, \\ México \\ \{mceron, etelvina, agarces,bbeltran\}@cs.buap.mx, jair@gmail.com
}

\begin{abstract}
Resumen. El propósito de este artículo es presentar el diseño de un sistema interactivo con escenarios de aprendizaje para apoyar a estudiantes con discapacidad auditiva de educación media superior en el área de biología. La metodología utilizada fue de prototipos y el diseño centrado en el usuario, lo cual permitió el uso del dispositivo natural del kinect ${ }^{\circledR}$ y la RA para dispositivos móviles como tabletas y Smartphone con Android. Finalmente se presentan los resultados obtenidos de una prueba piloto a un grupo focal de estudiantes con discapacidad auditiva, la experiencia de usuario y usabilidad del sistema.
\end{abstract}

Palabras claves: interfaces naturales de usuario, discapacidad auditiva, ambientes virtuales de aprendizajes.

\section{Design of learning scenarios with natural interfaces and augmented reality to support the inclusion of students with hearing disabilities in upper secondary education}

\begin{abstract}
The purpose of this paper is to present the design of an interactive system with learning scenes with augmented reality for helping to support hearing impaired students of high school in biology area. The methodology used was Prototypes and the Centered Design of the User, which allowed the use of the natural device of Kinect ${ }^{\circledR}$ and AR for mobile devices like tablets and smartphones with Android. Finally, it is presented the achieved results from a pilot test to a focal group of hearing impaired students, the user experience and the usability of the system.
\end{abstract}

Keywords: natural user interfaces, auditory disability, learning virtual environment. 


\section{Introducción}

La tecnología digital se ha convertido una herramienta importante para las personas con discapacidad, ya que les permiten acceder a diferentes entornos: recreación, educación, trabajo, comunicación e información. La Organización de las Naciones Unidas para la Educación, la Ciencia y la Cultura (UNESCO) afirma que la "inclusión se orienta a transformar los sistemas educativos para responder a la diversidad del alumnado y a una educación con igualdad de oportunidades: acceso, permanencia, participación y los logros de todos los estudiantes, con especial énfasis en aquellos que, por diferentes razones, están excluidos o en riesgo de ser marginados" [1]. Tal es el caso de personas con discapacidad, indígenas y niños. Es por eso que existe la necesidad de incidir e integrar a estudiantes con discapacidad en la educación media superior, logrando ser inclusivos mediante entornos de aprendizajes enriquecidos para todos. Una de las necesidades que se han identificado en la educación media superior para lograr la inclusión de esta población es la de propiciar espacios, aulas, desarrollar materiales didácticos, ayudas de software y recursos que puedan utilizarse en la modalidad presencial de forma accesible y permitan enriquecer el aprendizaje de los estudiantes.

El propósito de esta investigación es presentar el diseño de un sistema interactivo SIM's Anatomy con escenarios de aprendizaje accesibles por medio de Interfaces Naturales de Usuario mediante el uso del dispositivo del Kinect®, para facilitar la estimulación física y cognitiva, mediante uso de señas, movimientos y reconocimiento postural del cuerpo humano. Así también, el uso de escenarios en dispositivos móviles Smartphone o Tabletas con sistema Android integrando realidad aumentada para la representación de las actividades de aprendizaje e interacción, adaptándose a las necesidades y limitaciones de los usuarios para lograr el acceso al sistema, siendo atractivo y amigable acerca de temas relacionados con Tópicos de Biología: Cuerpo Humano, llamado esta versión de móviles como sistema AR'Anatomy. Este sistema permitirá apoyar el proceso aprendizaje y desarrollo de competencias disciplinares en los estudiantes con discapacidad auditiva mediante actividades de aprendizaje interactivas con RA.

El artículo está organizado como se indica a continuación: en la sección 2 se presenta la fundamentación y la revisión teórica del trabajo de las Interfaces Naturales de Usuario y experiencias de aprendizaje de realidad aumentada. La sección 3 se describe el análisis y el diseño del sistema, la arquitectura e implementación del sistema con las tecnologías de Kinect ${ }^{\circledR}$ y de los dispositivos móviles. En la sección 4 se muestran los resultados de la prueba piloto del grupo focal con el prototipo del sistema. Finalmente, se presenta las conclusiones y el trabajo a futuro de esta investigación.

\section{Estado del arte}

En esta sección se revisan aspectos de discapacidad auditiva, las interfaces naturales de usuario y la realidad aumentada, así como diferentes experiencias aplicadas al campo de la educación, los cuales aportan fundamentos para este trabajo de investigación. 
Diseño de escenarios de aprendizaje con interfaces naturales y realidad aumentada para apoyar ...

\subsection{Discapacidad auditiva e implicaciones educativas}

La discapacidad auditiva es la dificultad o imposibilidad que presenta la persona al utilizar el sentido del oído. En términos de la capacidad auditiva, se detectan dos conceptos relacionados, la hipoacusia y sordera. La primera se refiere a la pérdida auditiva de leve a moderada; no obstante, resulta funcional para la vida diaria. Por otra parte, la segunda es conocida como sordera se refiere a la pérdida auditiva de severa a profunda, donde la audición no es funcional para la vida diaria aun con auxiliares auditivos; la adquisición del lenguaje oral no se da de manera natural [2]. Los alumnos con este tipo de perdida utilizan principalmente el canal visual, para recibir la información, aprender y comunicarse. Por lo que la Lengua de Señas es la lengua natural de las personas con esta condición y que requieren estrategias específicas por los docentes para ser atendidos en los distintos niveles educativos. Por consiguiente, la tecnología y las interfaces naturales con distintos dispositivos ofrecen una gama de posibilidades para apoyar y lograr una mayor interacción con estudiantes con tal discapacidad. Los grados de pérdida auditiva según Villalba [3] se muestran en la Tabla 1. Es importante reconocer las implicaciones que conlleva el reconocimiento de sonidos, la lengua de señas y comunicación, lo que hace necesario ciertas recomendaciones para el docente en cada grado de pérdida auditiva y que debe propiciar estrategias para el alumno.

Tabla 1. Grados de pérdida auditiva y recomendaciones educativas.

\begin{tabular}{|c|c|c|}
\hline $\begin{array}{l}\text { Pérdida } \\
\text { Auditiva }\end{array}$ & Implicaciones & Recomendaciones \\
\hline $\begin{array}{c}\text { Leve } \\
(20 \mathrm{a} 40 \mathrm{~dB})\end{array}$ & $\begin{array}{l}\text { No captan fragmentos con "s o t" o } \\
\text { una voz débil. Se distraen con } \\
\text { facilidad y requieren se les repita lo } \\
\text { que escuchan. }\end{array}$ & $\begin{array}{l}\text { Apoyarse con estrategias visuales con } \\
\text { un orden lógico y secuenciado. } \\
\text { Repetir puntos principales. }\end{array}$ \\
\hline $\begin{array}{c}\text { Moderada } \\
(41 \text { a } 70 \mathrm{~dB})\end{array}$ & $\begin{array}{l}\text { Solo escucha con voz fuerte. } \\
\text { Se apoyan en la lectura de labio } \\
\text { facial. Usan lenguaje tardío y } \\
\text { fonemas. Problemas en su } \\
\text { estructuración del lenguaje y bajo } \\
\text { vocabulario. }\end{array}$ & $\begin{array}{l}\text { Aplicar técnicas de colaboración en } \\
\text { grupos pequeños. } \\
\text { Utilizar ayudas técnicas digitales y } \\
\text { permitir el manejo de } \\
\text { comunicadores visuales y parlantes. }\end{array}$ \\
\hline $\begin{array}{c}\text { Severa } \\
(71 \text { a } 90 \mathrm{~dB})\end{array}$ & $\begin{array}{l}\text { Presentan problemas del lenguaje y } \\
\text { se aíslan de las personas. Requiere el } \\
\text { aprendizaje de Lenguaje de Señas o } \\
\text { interpretación de labio facial }\end{array}$ & $\begin{array}{l}\text { Manejo de Lenguaje de Señas } \\
\text { Ayudas técnicas digitales } \\
\text { Materiales audiovisuales } \\
\text { Estar siempre de frente al alumno } \\
\text { Trabajar en pareja. }\end{array}$ \\
\hline $\begin{array}{c}\text { Profunda } \\
\text { (más de } 90 \mathrm{~dB} \text { ) } \\
\text { y } \\
\text { Sordera Total }\end{array}$ & $\begin{array}{l}\text { Falta de un código de comunicación. } \\
\text { Confía más en lo visual y debe } \\
\text { estimular la percepción del tacto y } \\
\text { visual. No escuchan. } \\
\text { Voz distorsionada o sonidos } \\
\text { guturales emiten }\end{array}$ & $\begin{array}{l}\text { Uso de Lenguaje de Señas } \\
\text { Estrategias del Bilingüismo } \\
\text { Evitar discriminaciones } \\
\text { Utilizar ayudas técnicas digitales y } \\
\text { dispositivos con mayor interacción } \\
\text { visual. }\end{array}$ \\
\hline
\end{tabular}




\subsection{Interfaces naturales $\mathbf{y}$ aplicaciones}

Las interfaces naturales de usuario (NUI) son aquellas en las que se interactúa con un sistema, aplicación, etc., sin utilizar sistemas de mando o dispositivos de entrada de las GUI (Interfaces de Usuario Graficas), como sería el ratón, teclado alfanumérico, joystick, etc., y en su lugar, se hace uso de movimientos gestuales tales como las manos o el cuerpo que fungen como el mismo mando de control, algunos ejemplos de estos dispositivos naturales son: kinect sensor, leap motion controller, myo gesture control armband y Google Glass. En esta investigación nos enfocamos al dispositivo Kinect®.

\subsubsection{Dispositivo Kinect}

Microsoft Kinect Sensor, es un dispositivo que integra una gran cantidad de sensores para detectar movimiento corporal del usuario, al mismo tiempo captura el sonido permitiendo la interacción utilizando instrucciones de voz [11]. Es un sensor de bajo costo que permite reconocer los movimientos del cuerpo y del rostro del usuario eliminando así el uso de controles físicos. Se compone de 2 sensores de profundidad 3D, cámara RGB, arreglo de micrófonos, y un motor automático que ajusta la base. Reconoce hasta 20 puntos del cuerpo como son la cabeza, hombros, manos, etc. y se programa SDK (Software Development Kit) en C/C++, C\#, VB y librerías como OpenCV. Por lo que, como medio de reconocimiento de movimiento, de las manos y algunas señas específicas, se logra ejercitar procesos cognitivos y permitir que la persona con discapacidad auditiva utilice las interfaces naturales como un código de comunicación con los escenarios de aprendizaje [4].

\subsubsection{Experiencias con interfaces naturales}

En el proyecto Mudra [5], su objetivo fue utilizar como dispositivos unos guantes con marcadores de colores y un sensor de profundidad para simular las operaciones del dispositivo del ratón en el uso del sistema operativo. Por otra parte, en el área de la salud, un estudio propuesto para analizar y realizar un seguimiento sobre las posturas y movimientos que realizan los usuarios al colocarse en diversas posiciones y acciones motrices usando Kinect ${ }^{\circledR}$ para mejorar su salud física y mental [6]. Así también, en otra investigación, el uso de una interfaz natural como el Kinect ${ }^{\circledR}$ con carácter rehabilitador se ha utilizado en varios usuarios con problemas motrices en el que demuestran que el uso de interfaces naturales puede ayudar a la rehabilitación de personas con problemas de carácter motriz y aumenta la mejora notablemente frente a otros métodos de rehabilitación convencionales [7].

Las Nuevas Tecnologías (NT) cumplen dos funciones básicas especialmente vinculadas con el aprendizaje: la mediación cognitiva y la aceleración de la percepción estímulos sensoriales. Un escenario de aprendizaje apoyado en la tecnología debe ser enriquecido para incrementar la eficiencia e interactividad con los contenidos de aprendizaje, ya que la tecnología propicia la interacción de alto nivel y promueve el desarrollo de habilidades del pensamiento crítico, creativo y manejo espacial [8]. 
Diseño de escenarios de aprendizaje con interfaces naturales y realidad aumentada para apoyar ...

\subsection{Realidad Aumentada}

La Realidad aumentada (RA) concebida como "la generación de imágenes nuevas a partir de la combinación de información digital en tiempo real y el campo de visión de una persona" [9]. La RA requiere de componentes como el hardware: computadora o un dispositivo móvil, una pantalla, una cámara y un marcador (geolocalización, reconocimiento de imágenes) y el software siendo frameworks de RA, en dispositivos móviles con Android se puede utilizar Vuforia, ARToolKit entre otros. La RA se puede presentar de dos formas: reconociendo una imagen marcadora o mediante un punto de localización geográfica, por eso al señalar se requiere que un marcador brinde las dos opciones. Cuando se utiliza un marcador, básicamente se asocia un modelo virtual en tercera dimensión a un objeto físico; cuando se usa la localización, en lugar de reconocer un marcador, se asigna información digital a un grupo de coordenadas geográficas.

Los procesos que se realiza la RA por medio de un marcador comienza por una cámara que muestra una señal de video en tiempo real, la señal es digitalizada e interpretada por el programa que, a su vez, identifica el marcador y lo asocia con el contenido digital asignado a él y, finalmente, el contenido digital es reproducido dentro del marco de la señal de video a través de la pantalla del dispositivo móvil o el monitor de la computadora [10]. En este escenario, la realidad aumentada puede ayudar a los estudiantes a contextualizar su aprendizaje, ya que la creación de contenidos altamente interactivos basados en realidad aumentada apoya el proceso de aprendizaje, donde se utilizó el reconocimiento de una imagen marcadora para los contenidos interactivos.

\section{Análisis y diseño del prototipo}

Para el diseño se utilizó el Diseño Centrado en el usuario (DCU) y la metodología de prototipos Se realizaron entrevistas para la definición de los requerimientos de los escenarios de aprendizaje en el área de biología del "Sistema circulatorio cardiovascular", enfocándose en apoyar el desarrollo de las competencias disciplinares, las cuales son: 1) Identifica y localiza los principales órganos que componen el aparato circulatorio, 2) Analiza su funcionamiento básico, 3) Reconoce algunas enfermedades comunes del aparato circulatorio y de la sangre y 4) Detecta como prevenirlas.

Para el análisis y el diseño del sistema se determinaron los Casos de Uso del sistema en UML. El sistema permite identificar dos usuarios, son:

- Usuario Docente: Puede realizar consultas generales del seguimiento de estudiantes con respecto a los contenidos, actividades y evaluación del tema.

- Usuario Alumno con Moderada/Severa: Consulta los contenidos y materiales de información, al realizar el diagnóstico, el sistema adapta las interfaces y presenta los contenidos que requiere aprender y propone una serie de actividades lúdicas para el desarrollo de las competencias del sistema circulatorio cardiovascular (ver figura 1). 


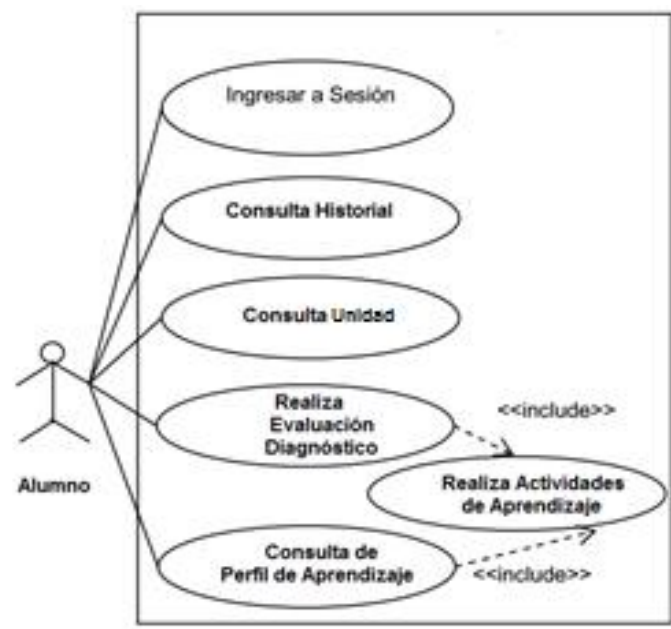

Fig. 1. Diagrama de caso de uso del alumno

Así también, se definieron los storyboards, el diseño de interfaces de usuario donde se muestra los temas de cada unidad, los elementos de apoyo (imágenes), en esta sección se podrá navegar con el Kinect y la detección de la mano para seleccionar una imagen o texto, como se muestra en la Figura 2.
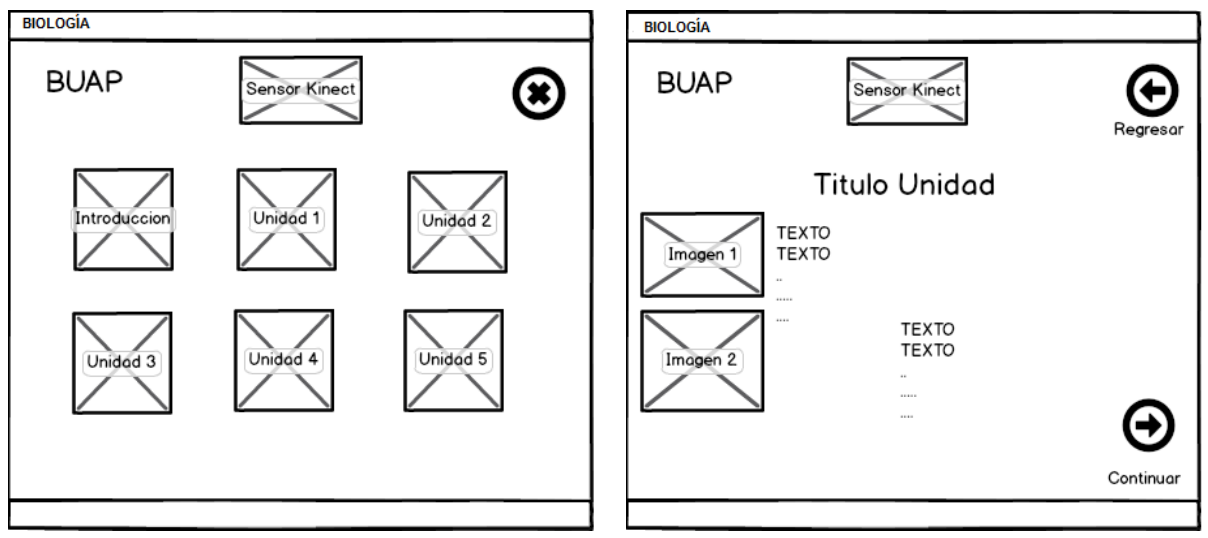

Fig. 2. Diseño de unidades del sistema

En la Figura 3, muestra el diseño de pantalla del Test por unidad la cual contendrá cuatro preguntas que se evaluaran y al terminar se presenta una ventana (modal) con el resultado obtenido, teniendo el formato de exámenes del Programa para la Evaluación Internacional de los Alumnos (PISA) [11]. Las preguntas de ciencias de PISA intentan evaluar hasta qué punto los alumnos aplican algún tipo de pensamiento científico a las situaciones que puedan encontrarse en sus vidas cotidianas y se distinguen tres dimensiones para la evaluación de las ciencias: conceptos, procesos y situaciones. 


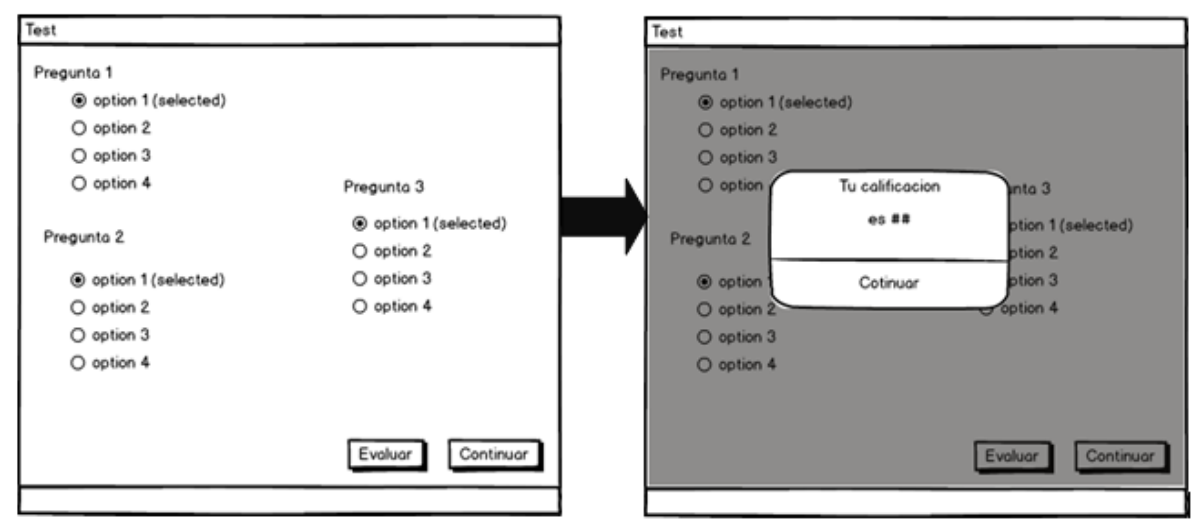

Fig. 3. Vista de evaluación de test formato PISA

Para la arquitectura del sistema, se identificaron elementos para el reconocimiento de de movimientos corporales mediante el Kinect, se elaboraron archivos y/o scripts en C++ y C\# [11]. Por otra parte, Vuforia es un SDK para dispositivos móviles que permite la creación de aplicaciones RA, proporciona (API) en C++ y Java, es compatible con el desarrollo nativo para iOS y Android, a la vez permitiendo el desarrollo de aplicaciones de RA, fáciles de transportar a ambas plataformas y el manejo compatible con dispositivos móviles como el iPhone, iPad, teléfonos y tabletas con una conexión a la base de datos MySql para la implementación y control de los usuarios e imágenes.

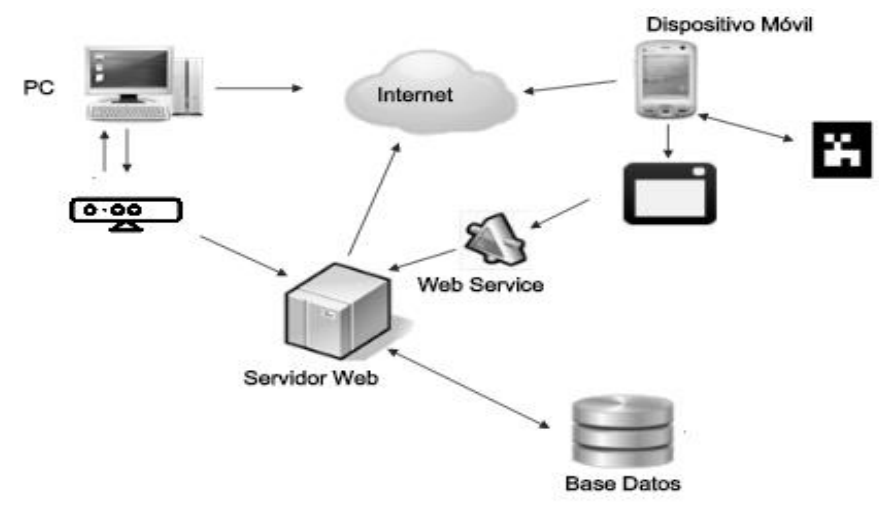

Fig. 4. Arquitectura del sistema.

\section{Desarrollo y pruebas del sistema}

El desarrollo del sistema Sim's Anatomy con el dispositivo Kinect® se programó en Greenhouse SDK en plataformas iOS, en C++ que permite la interacción a través de la 
entrada de reconocimiento de movimientos, como se muestra en la Figura 5. La librería G-speak ${ }^{\mathrm{TM}}$ permitió el manejo de múltiples pantallas de dispositivos móviles y en red. $\mathrm{Su}$ forma de trabajar se asemeja a una estructura cliente-servidor, la conexión se hace de manera local, mediante la dirección IP de la computadora. En cuanto al servidor (elegido); es necesario tener dos procesos ejecutandose: pool-tcp-server y pool-serverzeroconf-adapter, presentando extensiones a otros dispositivos.
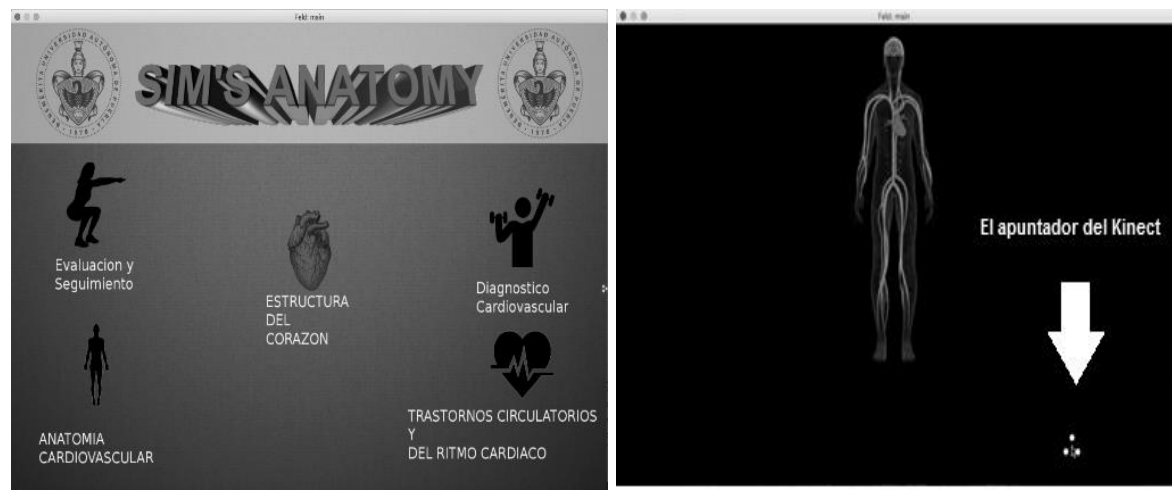

Fig. 5. Pantallas del sistema con uso del kinect.

Los estudiantes con discapacidad visual utilizan otros sentidos para la percepción, como el tacto y la vista, lo que fue considerado en el sistema para estimular estos dos sentidos e incluso el auditivo dependiendo del tipo de discapacidad auditiva del usuario. Por lo cual, el sistema se diseñó para lograr una interacción basada en gestos (movimientos o posicionamiento) y representación visual logrando una mayor interacción con el sistema y proporcionando una estimulación física y cognitiva para el desarrollo de las competencias de los estudiantes.
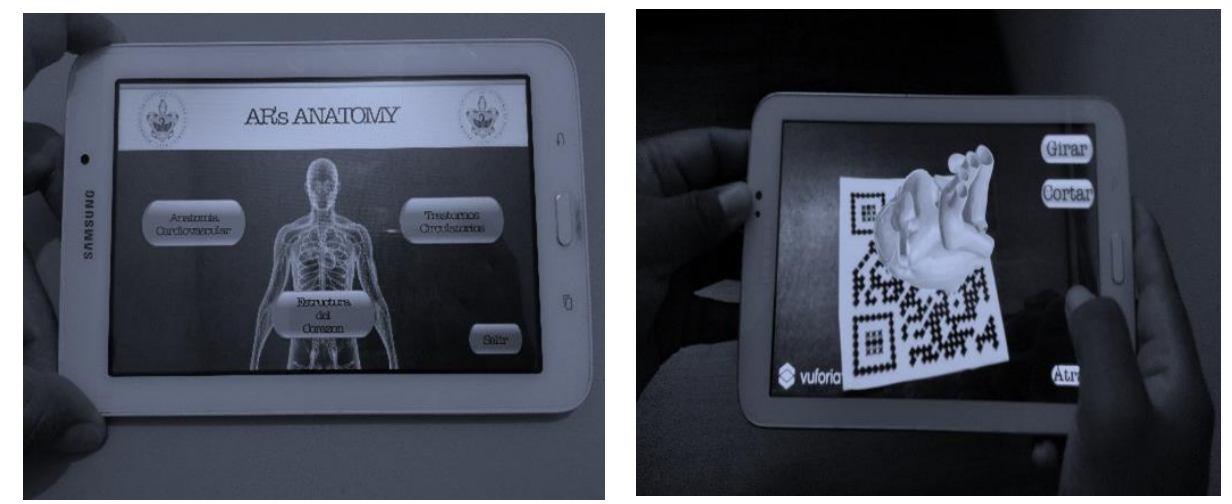

Fig. 6. RA del sistema circulatoria en Tablet con Android.

Para los contenidos enriquecidos con RA, versión dispositivo móvil la extensión del sistema conocida como AR's Anatomy se desarrolló con Vuforia en Unity y C++ con 
Android. Las actividades de exploración mediante RA, basada en marcadores y en un sistema de coordenadas, permitió contenidos interactivos en 3D, ver Figura 6.

El sistema considera tres niveles de desempeño de las competencias, la cuales son: 1) Iniciada, 2) En Proceso y 3) Desarrollada. De acuerdo al perfil pueden ser: Principiante, Intermedio y Avanzado. Para evaluar los contenidos se maneja en tres puntajes: 1) Bajo, menor a la media, 2) Regular, dentro de la media y 3) Alto, superior a la media, lo cual permite llevar el seguimiento académico del proceso de aprendizaje. El prototipo del sistema fue piloteado con un grupo focal de 6 estudiantes con discapacidad auditiva, de moderada-sordera total, los cuales pertenecen a educación media superior.

Con respecto a las pruebas de funcionalidad se aplicó la técnica de inspección con la experiencia de usuario. Para lo cual se presentaron tres posibles escenarios para los usuarios finales, en el uso de las interfaces naturales con el Kinect y con el dispositivo móvil con RA que a continuación se describen:

- Situación 1: Al estudiante con discapacidad moderada se le dio una breve explicación del uso del sistema y solo se le acompañó al inicio de la actividad.

- Situación 2: Al estudiante con discapacidad profunda se le explicó el uso del sistema y se le acompañó en la realización de algunas actividades.

- Situación 3: Al estudiante con discapacidad sordera total se le explicó el uso del sistema y se le acompañó en la realización de todas las actividades.

Para cada una de las situaciones los usuarios debieron cumplir ciertas tareas, con la finalidad de comprobar el funcionamiento del uso del sistema.

- Tarea 1: Poder ser detectado por el dispositivo del Kinect ${ }^{\circledR}$ y registrar su perfil.

- Tarea 2: Encontrar el menú de navegación y seleccionar una opción.

- Tarea 3: Utilizar los escenarios de aprendizaje y recorrerlos mediante el dispositivo natural del Kinect®, comandos de señas y movimientos corporales.

- Tarea 4: Activar el dispositivo móvil e interactuar con el sistema, seleccionando contenidos de RA.

- Tarea 5: Realizar las autoevaluaciones (diagnósticos) y reconocer su nivel de competencia.

Después de realizar las pruebas y de haber asignados solo a dos estudiantes por cada situación, se observó que los estudiantes con una breve explicación y acompañamiento al inicio (Situación 1), el desempeño fue en promedio del $94.8 \%$ del cumplimiento de las tareas, mientras que los usuarios que se les apoyo en algunas actividades (Situación 2) fue del $90 \%$, y para los estudiantes con sordera total (Situación 3), lograron realizar las tareas en un $92.8 \%$, lo cual representa una interfaz natural intuitiva, sencilla y agradable para los estudiantes, mientras los contenidos enriquecidos en dispositivos móviles les facilita el acceso a la información, interacción y motivación . Por otra parte, el desarrollo de las competencias se enfatiza en las competencias 1 y 2 , que se muestra en la Tabla 2.

Finalmente, se aplicó una encuesta de satisfacción, Valoración del Software [12], la cual evalúa siete criterios: Navegación, Interactividad, Inmersión, Usabilidad, Creatividad, Efectividad y Calidad, con una escala de 1 a 5 , cuyo promedio obtenido 
fue de 4.6 (92\%), lo que nos indica que la satisfacción del estudiante es alta para poder desarrollar sus competencias y un aprendizaje significativo, debido al uso de las interfaces naturales y de la RA como se muestra en la siguiente Figura 7.

Table 2. Nivel de las competencias

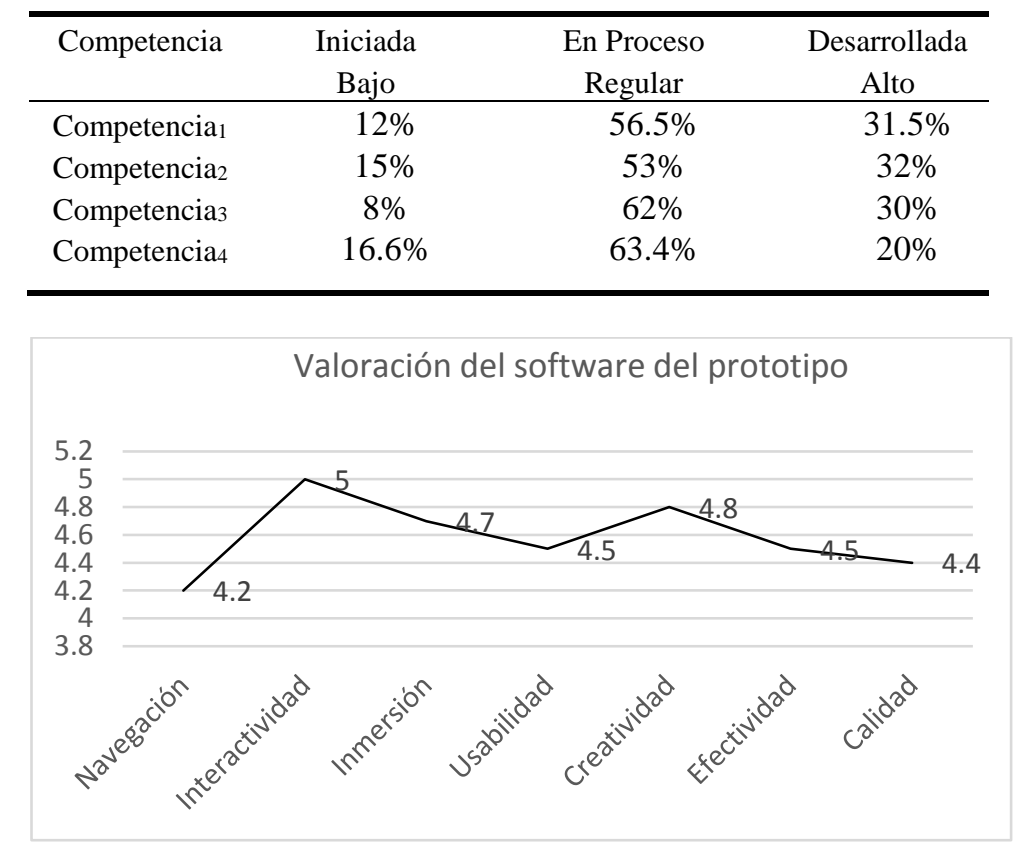

Fig. 7. Resultados de satisfacción del Prototipo

\section{Conclusiones y trabajos futuros}

Una de las principales contribuciones del sistema como prototipo, es la aportación a la inclusión de los estudiantes con discapacidad auditiva, propiciando experiencias de aprendizaje significativas apoyadas del uso de interfaces naturales y realidad aumentada, lo que permite el proceso de adquisición y desarrollo de las competencias disciplinares de la materia de biología y fortalecer el perfil de los estudiantes con alguna discapacidad auditiva.

El objetivo de integrar realidad aumentada a contenidos interactivos, ha permitido proponer que las nuevas tecnologías aplicadas en la educación y en otras áreas, es una opción que se debe implementar en el diseño y desarrollo de materiales educativos interactivos para estudiantes y ayudar al mismo tiempo el desarrollo de habilidades digitales en distintas plataformas y dispositivos. La perspectiva de este trabajo es realizar la evaluación de la accesibilidad y usabilidad del sistema propuesto ampliando la muestra de estudio y poder proveer a los alumnos con discapacidad auditiva materiales y herramientas digitales que puedan enriquecer el proceso de aprendizaje en distintas asignaturas logrando adaptaciones a exámenes, materiales, herramientas de 
Diseño de escenarios de aprendizaje con interfaces naturales y realidad aumentada para apoyar ...

estudio para apoyar la vida académica y promover la inclusión de los estudiantes con alguna discapacidad en el uso de las tecnologías digitales logrando disminuir los limitantes y barreras de la brecha digital que se presentan al interior de las instituciones educativas.

\section{Referencias}

1. Organización de las Naciones Unidas para la Educación, La Ciencia y la Cultura (UNESCO): Educación para Todos en las Américas. Marco de Acción Regional, República Dominicana (2000)

2. Organización Mundial de la Salud (OMS): Resumen de Informe Mundial de Discapacidad, (2001)

3. Villalba, A.: La pérdida de audición. Tipos de sordera y consecuencias que se derivan para la educación, Consellería de Cultura, Educación y Ciencia. Generalitat, Valenciana (1996)

4. Valli, A.: Notes on natural interaction (2005)

5. Anki, D., Yogesh, B., Abin, A., Rekha, S: Project MUDRA: Personalization of Computers using Natural Interface. International Journal of Computer Applications, Volume 54, Number 17, pp. 42-46 (2012)

6. Clark, R., Pua, Y., Fortin, K., Ritchie, C., Webster, K., Denehy, L., Bryant, A. L.: Validity of the Microsoft Kinect for assessment of postural control. Gait and Posture, Volume 36, Issue 3, pp. 372-377 (2012)

7. Chang, Y., Chen, S., Huang, J.: A Kinect-based system for physical rehabilitation: A pilot study for young adults with motor disabilities. Research in Developmental Disabilities, Volume 32, Issue 6, pp. 2566-2570 (2011)

8. Herrera, M.: Las Nuevas Tecnologías en el Aprendizaje Constructivo. Revista Iberoamericana de Educación (2004)

9. Johnson, L., Adams, S., Gago, D., García, E., Martín, S.: NMC perspectivas tecnológicas: educación superior en América Latina 2013-2018. Un análisis regional del informe Horizon del NMC. Austin, Texas: The New Media Consortium (2013)

10. Badilla, M., Sandoval A.: Realidad Aumentada como Tecnología Aplicada a la Educación Superior: Una Experiencia en Desarrollo. Universidad Estatal a Distancia (2016)

11. Microsoft Kinect Sensor (2015)

12. Acuña, A., Romo, M.: Diseño Instruccional Multimedia. Pearson Education, México (2011) 